\title{
BIFURCATION ANALYSIS OF STABILITY OF TRIANGULAR EQUILIBRIUM POINTS IN THE ELLIPTIC RESTRICTED PROBLEM OF THREE BODIES
}

\author{
T. Usha ${ }^{1 \S}$, A. Narayan ${ }^{2}$ \\ ${ }^{1,2}$ Bhilai Institute of Technology \\ Durg, INDIA
}

\begin{abstract}
This paper analyses the local bifurcation of linear stability of motion near the triangular equilibrium points in the neighborhood of parametric resonance frequency $\omega_{2}=\frac{1}{2}$. The periodic linear Hamiltonian system considered is dependent on small parameter $v$ (true anamoly). The system experiences the resonance at $\omega_{2}=\frac{1}{2}$ for circular and elliptic orbits, respectively. The Hamiltonian is made independent of time using canonical transformations. It is found that the values of mass ratio, $\mu$ are less than the critical value $\mu_{c}$ in resonant case, for both orbits. The boundary of the stability region are found in form of equations for both orbits, circular and elliptic (for small values of $e$ ). The regions of stability and instability are plotted in the graphs. There is a shift in the bifurcation points of mass ratio, $\mu$ from the critical value $\mu_{c}$ as a result of increase in values of radiation pressure $(\delta)$, triaxiality parameters $\left(\sigma_{1}\right.$ and $\left.\sigma_{2}\right)$.
\end{abstract}

AMS Subject Classification: $70 \mathrm{~F} 15$

Key Words: celestial mechanics, elliptical restricted three body problem, Lagrangian points, stability, oblateness, parametric resonance, bifurcation, local bifurcation

\section{Introduction}

The restricted three body problem has been widely studied by many researchers

Received: December 29, 2016

Revised: $\quad$ April 7, 2017

Published: July 14, 2017

(c) 2017 Academic Publications, Ltd. url: www.acadpubl.eu

$\S_{\text {Correspondence author }}$ 
due to its wide application to the celestial mechanics. The system under consideration consists of two finite bodies (known as primaries) moving about their common center of mass, having no influence on each other. The third body is influenced by both the primaries. The primaries may describe either circular or elliptic path. If the system performs motion about circular orbit, then it is circular restricted three body problem otherwise it is elliptic restricted three body problem. The elliptical restricted three body problem is a generalization of the classical restricted three body problem. The main difference between circular and elliptic problem is that the equilibrium points in circular are independent of time while for elliptic problem it is dependent on time. The Hamiltonian of the problem is also function of time. In the classical problem the primaries were considered to be perfectly spherical in shape because of homogeneous distribution of mass. But, in practice, some planets are oblate. This led to study of generalized restricted three body problem, by considering the shapes of bodies. To describe and analyze more systems in stellar dynamics, primaries were also considered as source of radiation. The elliptic restricted three body problem has been described in considerable detail by various authors, see [5], [23], [13], [8], [15], [14], [3]. In their study, authors have studied about the linear as well as the non-linear stability of motion near the triangular libration points. [11], used perturbation scheme to study the infinitesimal motions about triangular points in the elliptic restricted three body problem and derived fourth order analytical expressions. In celestial mechanics, the resonance phenomena occurs when two orbiting bodies exert a regular, periodic gravitational influence on each other. This is because their orbital periods are related by a ratio of two small integers. Orbital resonance affects the two bodies by altering or constraining the orbits as a result of mutual gravitational attraction. Resonance may involve one or any combination of the orbit parameters (e.g. eccentricity versus semi major axis, or eccentricity versus orbital inclination). It acts on any time scale from short term, commensurable with the orbit periods, to secular, measured in $10^{4}$ to $10^{6}$ years. Resonance also leads to either long-term stabilization of the orbits or be the cause of their destabilization. The stability of motion near the Lagrangian points in resonance case has also been a subject of investigation, and has been studied by different authors. The resonance case was studied in detail by [13]. The author established that the triangular libration points are stable in the first approximation for all ratios of the masses in the stability range, except for some specific ratios for which they are unstable. The author also studied the stability of the triangular libration points for the Sun-Jupiter system and proved that the plane three-body solution will be stable for most initial conditions. In [25] a semi-analytic perturbation theory for the motion near $3 / 1$ commensurability in 
the planar restricted three body problem was proposed and a new criterion was given for the existence of large-scale chaotic behavior. The restricted problem of three bodies when one of the primary is radiating was studied in [17]. They established the existence of Lagrange's particular solutions and investigated their non-linear stability in the resonance cases. In [6], a completely integrable dynamical system representing the averaged motion of an asteroid moving in first order resonance with Jupiter placed in an elliptic orbit was derived. In[12], the stability of triangular libration points for doubly photogravitational elliptic restricted problem of three bodies under the presence of resonances as well as under their absence was derived and the condition of stability was found. In[9], the elliptic restricted three body problem was analyzed and computed four 3 : 1 resonant families of periodic orbits, modeling Sun-Jupiter asteroid system. Later, in [10], the resonant structure of restricted three body problem for both circular and elliptical orbits, modeling Sun-Jupiter asteroid was studied. The author analysed three different resonances $2: 1,3: 1$ and $4: 1$ and concluded that the bifurcation points shift from the classical problem. Later, in [21] the non-linear stability of $L_{4}$ in the restricted three body problem by taking bigger primary as oblate was studied and three different range for critical values of $\mu$ were found. [26], analysed the stability of equilibrium points and demonstrated that parametric resonance causes instability of equilibrium points. [4] studied the non-linear stability of triangular libration points in the restricted three body problem with bigger oblate primary and concluded that the system is stable for resonant case $\omega_{1}=3 \omega_{2}$ and unstable for $\omega_{1}=2 \omega_{2}$. [14] is the extension of their previous study by investigating the case of parametric resonance for elliptic restricted three body problem. In the same year, in [24] and [20] the linear stability of the equilibrium points in the elliptic restricted three body problem was analyzed.

In general a resonance can arise when there is a simple numerical relationship between frequencies or periods [18]. Parametric resonance arises when external frequency is twice the natural frequency. It is a resonance phenomena different from normal resonance because it is an instability phenomena. This may cause bifurcation of equilibrium points from the classical problem. A bifurcation occurs when a small change in the parameter values of a system causes sudden change in its behavior. It can be classified as local and global bifurcations respectively, depending on the stationary points. The local bifurcation can be analyzed through the changes in the stability property of equilibrium points or periodic orbits. Where as, global bifurcations occur when larger invariant sets of the system collide with the equilibrium points of the system. It cannot be depicted by the stability analysis of the equilibrium points. 
In the present paper we study and analyze the local bifurcation of linear stability of the triangular equilibrium points $L_{4,5}$ in the elliptic restricted three body problem in presence of parametric resonances. In this model, the bigger primary is a source of radiation and the smaller one is oblate and triaxial. This is achieved by the method given in [13] in which the Hamiltonian is made independent of time using canonical transformations. This model can easily be applied in the astrophysical applications for the study of many stellar systems.

The present paper is organized as follows: Section 1 gives the introduction; The equations of motion are described in Section 2. In Section 3 and its subsections, the analysis of stability is done, for the cases $e=0$ and $e \neq 0$. Finally, discussions and conclusions are drawn in Section 4.

\section{Equations of Motion}

The differential equations of motion of the infinitesimal mass in the elliptic restricted three body problem under radiating and triaxial primaries in the barycentric, pulsating and rotating, non-dimensional coordinates are given in the following equation (1). The notations in principle are taken from the book [23] with some minor modifications in the notations being done for adapting to the present model, which is given by:

$$
x^{\prime \prime}-2 y^{\prime}=\frac{1}{(1+e \cos v)}\left(\frac{\partial \Omega}{\partial x}\right) ; y^{\prime \prime}+2 x^{\prime}=\frac{1}{(1+e \cos v)}\left(\frac{\partial \Omega}{\partial y}\right) .
$$

where' denotes differentiation with respect to $v$, and

$$
\Omega=\frac{x^{2}+y^{2}}{2}+\frac{1}{n^{2}}\left[\frac{(1-\mu) q}{r_{1}}+\frac{\mu}{r_{2}}+\frac{\mu\left(2 \sigma_{1}-\sigma_{2}\right)}{2 r_{2}^{3}}-\frac{3 \mu\left(\sigma_{1}-\sigma_{2}\right) y^{2}}{2 r_{2}^{5}}\right]
$$

where,

$$
\begin{gathered}
n^{2}=1+\frac{3}{2}\left(2 \sigma_{1}-\sigma_{2}\right) ; r_{1}^{2}=(x+\mu)^{2}+y^{2} ; r_{2}^{2}=(x+\mu-1)^{2}+y^{2} \\
\phi=\frac{1}{1+e \cos v} ; \sigma_{1}=\frac{a^{2}-c^{2}}{5 R^{2}} ; \sigma_{2}=\frac{b^{2}-c^{2}}{5 R^{2}} ;
\end{gathered}
$$

Here, $\mu=\frac{m_{2}}{m_{1}+m_{2}}$, where, $m_{1}$ and $m_{2}$ are the masses of the bigger and smaller primaries positioned at $\left(x_{i}, 0\right), i=1,2 ; q$ is the radiation pressure, $q=1-\delta$; 
$\sigma_{i}<<1,(i=1,2)[16]$; and $a, b, c$ are semi axes and $R$ is the distance between the primaries; $r_{i}(\mathrm{i}=1,2)$ are the distances of the infinitesimal mass from the bigger and smaller primaries respectively; while $e$ is the eccentricity of the either primary around the other and $v$ is the true anomaly. Then the co-ordinates $(x, y)$ of the triangular equilibrium points $L_{4}$ and $L_{5}$ following [24] are:

$$
\begin{aligned}
x= & \frac{1}{2}-\mu-\frac{\delta}{3}+\left\{\frac{3}{8}-\delta+\frac{\mu}{2(1-\mu)}\right\} \sigma_{1}+\left\{\frac{-7}{8}+\frac{\delta}{2}-\frac{\mu}{2(1-\mu)}\right\} \sigma_{2} ; \\
y= & \pm \frac{\sqrt{3}}{2}\left[1+\frac{2}{3}\left\{\frac{-\delta}{3}+\left(\frac{-19}{8}-\delta+\frac{\mu}{2(1-\mu)}\right) \sigma_{1}\right.\right. \\
& \left.\left.+\left(\frac{15}{8}+\frac{\delta}{2}-\frac{\mu}{2(1-\mu)}\right) \sigma_{2}\right\}\right] .
\end{aligned}
$$

The perturbed Hamiltonian is given by:

$$
\begin{aligned}
H= & \frac{1}{2}\left(p_{x}^{2}+p_{y}^{2}\right)+\left(y p_{x}-x p_{y}\right)+\frac{e \cos v}{2(1+e \cos v)}\left(x^{2}+y^{2}\right)-\frac{1}{1+e \cos v} \\
& \times\left[\frac{1}{n^{2}}\left\{\frac{(1-\mu) q}{r_{1}}+\frac{\mu}{r_{2}}+\frac{\mu\left(2 \sigma_{1}-\sigma_{2}\right)}{2 r_{2}^{3}}-\frac{3 \mu\left(\sigma_{1}-\sigma_{2}\right) y^{2}}{2 r_{2}^{5}}\right\}\right] .
\end{aligned}
$$

The two equilibrium solutions are symmetrical, the nature of the motion near the two points will be the same. So, the motion will be analysed near one of the equilibrium points $L_{4}$. The perturbed system in the neighborhood of $L_{4}$ is given by shifting the origin, obtained by the change of variables at $L_{4}$ given as follows:

$$
x=\xi+q_{1} ; y=\eta+q_{2} ; p_{x}=p_{\xi}+p_{1} ; p_{y}=p_{\eta}+p_{2}
$$

The solution of (5) in the new variables are given by $q_{1}=q_{2}=p_{1}=p_{2}=0$, which is the equilibrium position. The Hamiltonian $H$ can be written in the form of $H_{k}$ as the sum of terms of the $k^{t h}$ degree homogeneous in the variables $q_{1}, q_{2}, p_{1}, p_{2}$, as:

$$
H=\Sigma_{0}^{\infty} H_{k}=H_{0}+H_{1}+H_{2}+H_{3}+H_{4}+\cdots \cdots
$$

where,

$$
H_{0}=H\left(\xi, \eta, p_{\xi}, p_{\eta}\right)=\text { constant and } H_{1}=0
$$

The second order Hamiltonian after simplification in form of general coordinates $\left(q_{i}, p_{i}\right)\{i=1,2\}$ is given by:

$$
H_{2}=\frac{1}{2}\left(p_{1}^{2}+p_{2}^{2}\right)+\left(p_{1} q_{2}-p_{2} q_{1}\right)+\frac{e \cos v}{2(1+e \cos v)}\left(q_{1}^{2}+q_{2}^{2}\right)
$$




$$
+\frac{1}{(1+e \cos v)}\left(q_{1}^{2} A-q_{1} q_{2} B-q_{2}^{2} C\right),
$$

where

$$
\begin{gathered}
A=\frac{1}{8}+\frac{\delta}{4}+\left(-\frac{21}{16}+\frac{3 \mu}{64}\right) \sigma_{1}+\left(\frac{27}{16}-\frac{99 \mu}{64}\right) \sigma_{2} \\
B=\frac{\sqrt{3}}{4}\left\{\frac{3}{4}-\frac{3 \mu}{2}-\frac{2 \delta}{3}+\left(\frac{7}{2}-\frac{21 \mu}{8}\right) \sigma_{1}+\left(\frac{-9}{2}+\frac{181 \mu}{8}\right) \sigma_{2}\right\} \\
C=\frac{5}{8}+\frac{\delta}{4}-\frac{3 \mu \delta}{4}+\left(-\frac{21}{16}+\frac{183 \mu}{64}\right) \sigma_{1}+\left(\frac{27}{16}-\frac{183 \mu}{64}\right) \sigma_{2} .
\end{gathered}
$$

\section{Stability of the System}

The second order Hamiltonian of the problem is given by (8). The Hamiltonian mainly depends on $e$. So, to analyse the stability considering the two cases. First, when $e=0$, i.e., the orbit is circular and the second one for small values of $e$.

\subsection{Stability in Circular Case}

When $e=0$, the expression (8) becomes the Hamiltonian for the circular problem upto $2^{\text {nd }}$ order terms given by:

$$
H_{2}=\frac{1}{2}\left(p_{1}^{2}+p_{2}^{2}\right)+\left(p_{1} q_{2}-p_{2} q_{1}\right)+\left\{A q_{1}^{2}-B q_{1} q_{2}-C q_{2}^{2}\right\} .
$$

The variational equation for the circular problem can be written as:

$$
\dot{p}_{i}=-\frac{\partial H_{2}}{\partial q_{i}} \text { and } \dot{q}_{i}=\frac{\partial H_{2}}{\partial p_{i}}
$$

Using the variational equation and with the help of canonical linear transformation, the characteristic equation is obtained as:

$$
\begin{aligned}
\lambda^{4}+\left\{1-\frac{45}{8} \mu \sigma_{1}+\right. & \left.\frac{21}{8} \mu \sigma_{2}\right\} \lambda^{2} \\
& +\frac{27}{4} \mu(1-\mu)\left\{1+\frac{2}{9} \delta+\left(\frac{71}{8} \sigma_{1}-\frac{181}{24} \sigma_{2}\right)\right\}=0 .
\end{aligned}
$$


Let $\lambda_{1}=i \omega_{1} ; \lambda_{2}=i \omega_{2}$. The equation (11) can be written as:

$$
\begin{aligned}
\omega^{4}-\left\{1-\frac{45}{8} \mu \sigma_{1}+\right. & \left.\frac{21}{8} \mu \sigma_{2}\right\} \omega^{2} \\
& +\frac{27}{4} \mu(1-\mu)\left\{1+\frac{2}{9} \delta+\left(\frac{71}{8} \sigma_{1}-\frac{181}{24} \sigma_{2}\right)\right\}=0 .
\end{aligned}
$$

So, the frequencies $\omega_{1}, \omega_{2}$ are given by:

$$
\begin{aligned}
\omega_{1,2}^{2}= & \frac{1}{2}\left[\left\{1-\frac{45}{8} \mu \sigma_{1}+\frac{21}{8} \mu \sigma_{2}\right\} \pm\left\{\left(1-\frac{45}{4} \mu \sigma_{1}+\frac{21}{4} \mu \sigma_{2}\right)\right.\right. \\
& \left.\left.-27 \cdot \mu \cdot(1-\mu)\left(1+\frac{2}{9} \delta+\frac{71}{8} \sigma_{1}-\frac{181}{24} \sigma_{2}\right)\right\}^{\frac{1}{2}}\right]
\end{aligned}
$$

For the stability of the equilibrium position, $\omega_{1,2}$ must be purely imaginary. Hence

$$
\left\{\left(1-\frac{45}{4} \mu \sigma_{1}+\frac{21}{4} \mu \sigma_{2}\right)-27 \cdot \mu \cdot(1-\mu)\left(1+\frac{2}{9} \delta+\frac{71}{8} \sigma_{1}-\frac{181}{24} \sigma_{2}\right)\right\} \geq 0
$$

Therefore, the region of stability in the first approximation can be written as:

$$
0<\mu<\frac{\left(1+\frac{45}{4} \sigma_{1}-\frac{21}{4} \sigma_{2}\right)-\sqrt{\frac{23}{27}}\left(1+\frac{4}{207} \delta+\frac{643}{46} \sigma_{1}-\frac{941}{138} \sigma_{2}\right)}{2}
$$

Thus, the value of $\mu$ responsible for stable equilibrium points is given by:

$$
\mu_{c}=0.0385205-0.008915 \delta-0.825675 \sigma_{1}+0.52175 \sigma_{2}
$$

Also, we have,

$$
\omega_{1}\left(\mu_{c}\right)=\omega_{2}\left(\mu_{c}\right)=\frac{1}{\sqrt{2}}
$$

The dependence of $\omega_{1}$ and $\omega_{2}$ on $\mu$ is given in the graph( $\left.3-4\right)$. In the region (15), the only parametric resonance $\omega_{2}=\frac{1}{2}$ is possible. The corresponding value of $\mu$ for $\omega_{2}=\frac{1}{2}$ is given by:

$$
\mu_{0}=0.028595-0.006547 \delta-0.2804 \sigma_{1}+0.231045 \sigma_{2}
$$

The above equation (17) gives the boundary of the stability region as well as the equation for local bifurcation, when the orbit is circular. The result is same as given in [11], when $\delta=0, \sigma_{1}=0, \sigma_{2}=0$. The is a shift in the bifurcation points as compared to classical problem. 


\subsection{Stability in Elliptical Case}

The Hamiltonian is given by equation (8). For small values of $e$, expanding the function $\mathrm{H}_{2}$ in the powers of $e$, upto first approximation, we have:

$$
\begin{aligned}
H_{2}=\frac{\left(p_{1}^{2}+p_{2}^{2}\right)}{2}+\left(p_{1} q_{2}\right. & \left.-p_{2} q_{1}\right)+\left(A q_{1}^{2}-B q_{1} q_{2}-C q_{2}^{2}\right) \\
& +e \cos v\left\{\left(\frac{1}{2}-A\right) q_{1}^{2}+B q_{1} q_{2}+\left(\frac{1}{2}+C\right) q_{2}^{2}\right\}
\end{aligned}
$$

Now, using the canonical transformation,

$$
\left[q_{1}, q_{2}, p_{1}, p_{2}\right]=\left[q_{1}^{\prime}, q_{2}^{\prime}, p_{1}^{\prime}, p_{2}^{\prime}\right] N
$$

where,

$$
N=\left[\begin{array}{cccc}
a_{1} & a_{1} c_{1} & -a_{1} c_{1} & a_{1}\left(1-\omega_{1}^{2} b_{1}\right) \\
a_{2} & a_{2} c_{2} & -a_{2} c_{2} & a_{2}\left(1-\omega_{2}^{2} b_{2}\right) \\
0 & a_{1} b_{1} & a_{1}\left(1-b_{1}\right) & a_{1} c_{1} \\
0 & -a_{2} b_{2} & -a_{2}\left(1-b_{2}\right) & -a_{2} c_{2}
\end{array}\right]
$$

and,

$$
a_{i}=\frac{1}{2} \sqrt{\frac{2 l_{i}}{\left|\omega_{i}^{2}-\frac{1}{2}\right|}} ; b_{i}=\frac{2}{l_{i}} ; c_{i}=\frac{-B}{l_{i}} ; l_{i}=\omega_{i}^{2}+2 C+1
$$

where $i=1,2$ and $A, B, C$ are same as given in equation (8), and $\omega_{i}$ are given by equation (13).

Now, rewriting (8) as:

$$
H_{2}=H_{2}^{(0)}+H_{2}^{(1)}
$$

where $H_{2}^{(0)}$ is the Hamiltonian independent of eccentricity and $H_{2}^{(1)}$ is the Hamiltonian containing first order approximation in $e$. So,

$$
\begin{aligned}
& H_{2}^{(0)}=\frac{\left(p_{1}^{2}+p_{2}^{2}\right)}{2}+\left(p_{1} q_{2}-p_{2} q_{1}\right)+\left(A q_{1}^{2}-B q_{1} q_{2}-C q_{2}^{2}\right) \\
& H_{2}^{(1)}=e \cos v\left\{\left(\frac{1}{2}-A\right) q_{1}^{2}+B q_{1} q_{2}+\left(\frac{1}{2}+C\right) q_{2}^{2}\right\}
\end{aligned}
$$

Now, using the transformations given in the (19), (20) and (21) in the above equation. The normalised Hamiltonian can be written as: 


$$
\begin{aligned}
H_{2}=\frac{1}{2}\left(p_{1}^{\prime 2}+\omega_{1}^{2} q_{1}^{\prime 2}\right)-\frac{1}{2}\left(p_{2}^{2}+\right. & \left.\omega_{2}^{2} q_{2}^{\prime 2}\right) \\
& +e \cos v\left[a q_{2}^{\prime 2}+b p_{2}^{\prime 2}+c q_{2}^{\prime 2} p_{2}^{\prime 2}+\ldots \ldots\right],
\end{aligned}
$$

where

$$
a=A a_{2}^{2}+B a_{2}^{2} c_{2}+C a_{2}^{2} c_{2} ; b=C a_{2}^{2} b_{2} ; c=-B a_{2}^{2} b_{2}-C a_{2}^{2} b_{2} c_{2} .
$$

here, dots denote the second order terms in $p_{1}^{\prime}$ and $q_{1}^{\prime}$, which are not taken into consideration for further calculations.

Now, taking the transformations of variables:

$$
q_{i}^{\prime}=(-1)^{i+1} \frac{\sqrt{2} \alpha_{i}}{\omega_{i}} \sin \omega_{i}\left\{v-(-1)^{i-1} \beta_{i}\right\} ; p_{i}^{\prime}=\sqrt{2} \alpha_{i} \cos \omega_{i}\left\{v-(-1)^{i-1} \beta_{i}\right\}
$$

and

$$
\omega_{2}=\frac{1}{2}+\epsilon, \quad|\epsilon| \ll 1
$$

The terms containing eccentricity are considered as perturbations in the Hamiltonian. So, using the above transformation of variables, the perturbation in the Hamiltonian is given by:

$$
\begin{aligned}
H_{2}^{(1)}= & e \cos v\left[\frac{2 a \alpha_{2}}{\omega_{2}^{2}} \sin ^{2} \omega_{2}\left(v-\beta_{2}\right)+2 b \alpha_{2} \cos ^{2} \omega_{2}\left(v-\beta_{2}\right)\right. \\
& \left.-\frac{c \alpha_{2}}{\omega_{2}} \sin 2 \omega_{2}\left(v-\beta_{2}\right)\right]
\end{aligned}
$$

Now, averaging the terms with finite frequencies between 0 to $2 \pi$, and eliminating $v$, and using the canonical transformations:

$$
\overline{\alpha_{1}}=\alpha_{1}, \overline{\beta_{1}}=\beta_{1} ; \overline{\alpha_{2}}=\alpha_{2}, \overline{\beta_{2}}=\left(\beta_{2}-2 \epsilon v\right)
$$

Hence, the non-periodic part of the perturbation is given as:

$$
\overline{H_{2}}=e\left\{U \cos 2 \omega_{2} \overline{\beta_{2}}-V \sin 2 \omega_{2} \overline{\beta_{2}}\right\} \alpha_{2}-2 \epsilon \alpha_{2}
$$

The integral of (27) is :

$$
e\left\{U \cos 2 \omega_{2} \overline{\beta_{2}}-V \sin 2 \omega_{2} \overline{\beta_{2}}\right\} \alpha_{2}-2 \epsilon \alpha_{2}=h_{1}=\text { constant }
$$

where,

$$
U=\left(\frac{b-4 a}{2}\right) ; V=c
$$


Therefore, the Hamiltonian $\overline{H_{2}}$ given by (27), have the solution:

$$
\overline{H_{2}}=e\left(U^{2}+V^{2}\right)^{\frac{1}{2}} \frac{h_{1}}{e\left(U^{2}+V^{2}\right)^{\frac{1}{2}}}
$$

satisfying the condition:

$$
\left|\frac{2 \epsilon}{e\left(U^{2}+V^{2}\right)^{\frac{1}{2}}}\right|<1
$$

i.e.,

$$
|\epsilon|<\frac{1}{2} e\left(U^{2}+V^{2}\right)^{\frac{1}{2}}
$$

The inequality (31) determines the region of parametric resonance in the $\mu-e$ plane in the neighborhood of the point $\mu_{0}$ corresponding to $\omega_{2}=\frac{1}{2}$. The boundary of the region in the first approximation, accurate upto $O\left(e^{2}\right)$, is given by :

$$
\mu_{0}-e A_{1}<\mu<\mu_{0}+e A_{1}
$$

where,

$$
A_{1}=\left.\frac{\left(U^{2}+V^{2}\right)^{\frac{1}{2}}}{2 \frac{d \omega_{2}(\mu)}{d \mu}}\right|_{\mu=\mu_{0}}
$$

Now, substituting all the required values in the above equation, the boundary of the region given by (32) of parametric resonance and local bifurcation about $\omega_{2}=\frac{1}{2}$ in the first approximation in $e$ has the form:

$$
\begin{aligned}
\mu= & 0.028595-0.006547 \delta-0.2804 \sigma_{1}+0.231044 \sigma_{2} \pm e(0.056416-0.01332 \delta \\
& \left.-0.432547 \sigma_{1}+0.387793 \sigma_{2}\right)
\end{aligned}
$$

The region of stability and instability of are plotted in the graphs (see Figures 1-2). When the orbit is taken elliptic the boundary region is by the equation (34). All the calculations are performed with the help of the software Mathematica and graphs have been plotted using Matlab.

\section{Conclusion And Discussions}

The analysis of local bifurcation of linear stability of the elliptic restricted three body problem with bigger primary as a source of radiation and the smaller one an oblate and triaxial body is done. The dependence of $\omega_{1}$ and $\omega_{2}$ on $\mu$ are given by equations (13 and shown in the graphs( $3-4)$. The value of mass ratio 
$\mu_{\text {critical }}$ for stable equilibrium position and local bifurcation is given by (16). The boundary of the stability and bifurcation regions are given by (17) in circular case and (34) gives stability region in first approximation in $e$, in elliptical case, respectively.

The region of stability and instability of the linear problem for small values of $e$ has been shown in graphs (Figure: 1- Figure: 2). The following are the points which are clear from the graphs (Figure: 1,Figure: 2):

(i) In the region with in the boundary, the triangular points are unstable while in the region outside the boundary curve, the necessary conditions of linear stability are satisfied.

(ii) The outer line of the graph(red) shows the classical boundary region. This region is decreasing as the value $\left|\sigma_{1}-\sigma_{2}\right|>0$ and $\delta$ increases. That is the stability of the system decreases with increasing values of the above parameters, $\delta, \sigma_{1}, \sigma_{2}$. This is due to the local bifurcation of the system.

The graphs shown by Figure: 3 and Figure: 4 shows the dependence of resonance frequency $\omega_{1,2}$ on mass ratio $\mu$. The following conclusions can be drawn from the graphs (Figure: 3, Figure: 4):

(i) From the graphs (Figure: 3, Figure: 4) it is clear that the value of $\omega_{1}$ decreases and the value of $\omega_{2}$ increases upto a value of $\mu$. This value of $\mu$ where both the frequencies $\omega_{1,2}$ intersect, and increases with increase in the value of $\delta$ and $\left|\sigma_{1}-\sigma_{2}\right|>0$.

(ii) The outer curve of the graphs(yellow) shows the curve when $\delta=0$ for values of $\left|\sigma_{1}-\sigma_{2}\right|>0$. The area of region under this curve goes on decreasing as the value of $\delta$ increases.

The radiation pressure and oblateness parameters produce a visible shift in the bifurcation points from the classical values which can be seen form graphs (Figure: 1, Figure: 2). These results are in confirmation with those given by [11], [9]. This study can further help to analyse the resonance cases and chaotic behavior of different celestial bodies. 


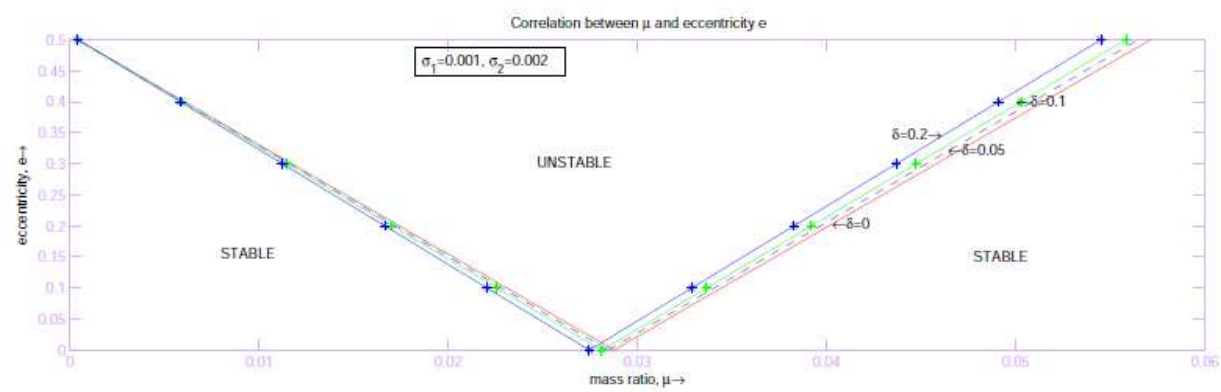

Figure 1: Correlation of mass ratio $\mu$ and eccentricity $e$

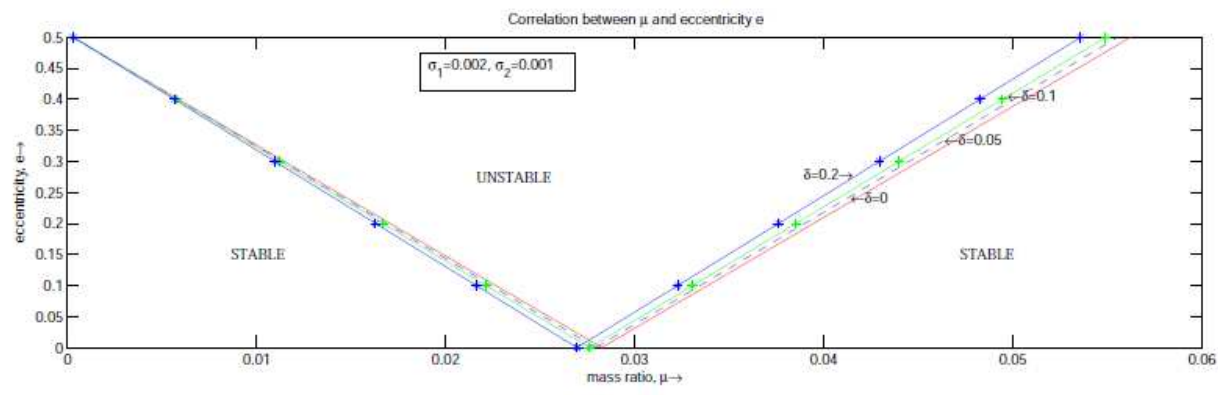

Figure 2: Correlation of mass ratio $\mu$ and eccentricity $e$

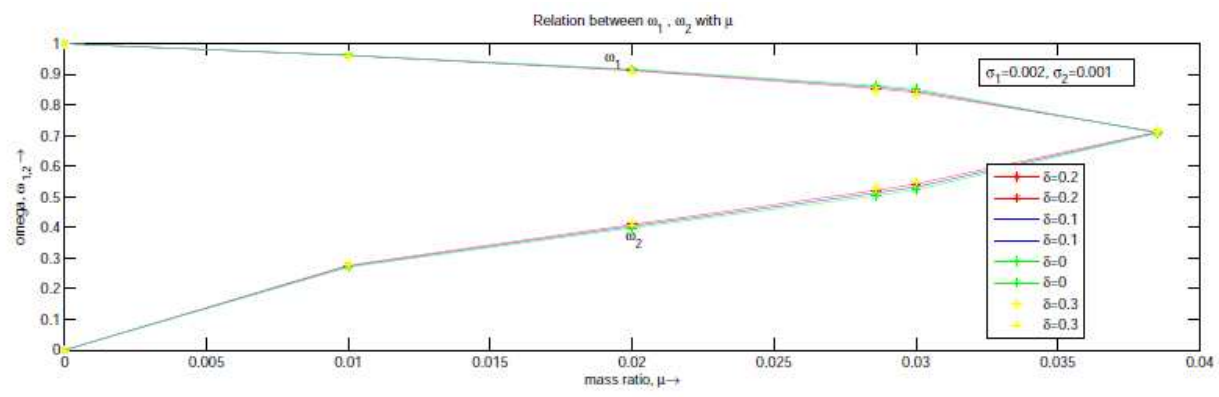

Figure 3: Correlation of mass ratio $\mu$ and eccentricity $e$ 


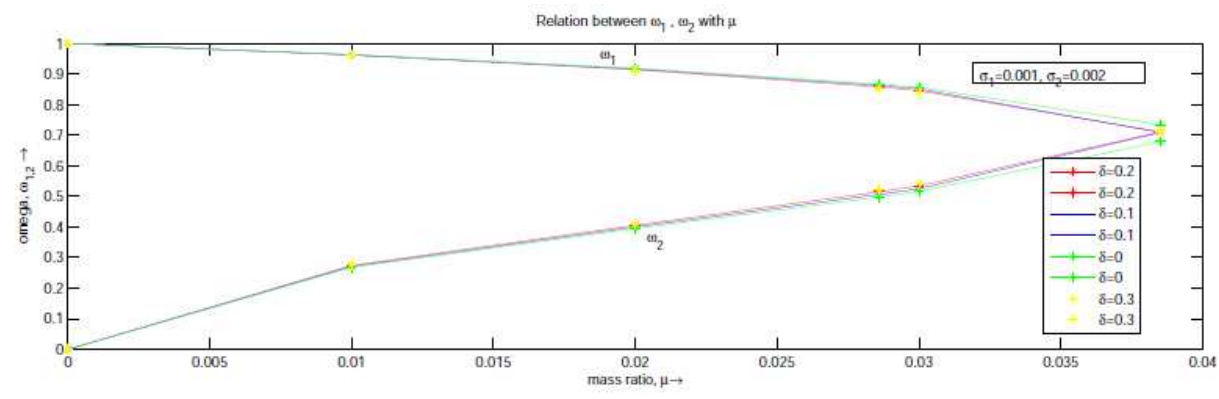

Figure 4: Correlation of mass ratio $\mu$ and eccentricity $e$

\section{References}

[1] V.I. Arnold, Stability of equilibrium position of a hamiltonian system of ordinary differential equations in general elliptic case, Doklady Akademii Nauk SSSR, 137, No. 2 (1961), 255.

[2] Bálint Erdi, Emese Forgács-Dajka, Imre Nagy, and Renáta Rajnai, A parametric study of stability and resonances around L 4 in the elliptic restricted three-body problem, Celestial Mechanics and Dynamical Astronomy, 104, No-s: 1-2 (2009).

[3] Arthur Bennett, Characteristic exponents of the five equilibrium solutions in the elliptically restricted problem, Icarus, 4, No. 2 (1965).

[4] Navin Chandra and Ranjeet Kumar, Effect of oblateness on the non-linear stability of the triangular liberation points of the restricted three-body problem in the presence of resonances, Astrophysics and Space Science, 291, No. 1 (2004).

[5] J.M.A. Danby, Stability of the triangular points in the elliptic restricted problem of three bodies, The Astronomical Journal, 69 (1964).

[6] S Ferraz-Mello, Averaging the elliptic asteroidal problem near a first-order resonance, The Astronomical Journal, 94 (1987), 208-212.

[7] E.A. Grebnikov, Nauka, Moscow Revised, 1986.

[8] E.A. Grebnikov, Soviet Astronomy, 8, No. 3 (1964), 451.

[9] John D. Hadjidemetriou, The elliptic restricted problem at the 3: 1 resonance, Celestial Mechanics and Dynamical Astronomy, 53, No. 2 (1992).

[10] John D. Hadjidemetriou, Resonant motion in the restricted three body problem, Qualitative and Quantitative Behaviour of Planetary Systems, Springer Netherlands (1993), 201-219.

[11] A.A. Kamel and A.H. Nayfeh, Stability of the triangular points in the elliptic restricted problem of three bodies, AIAA Journal, 8, No. 2 (1970).

[12] Vijay Kumar, and R.K. Choudhry, Nonlinear stability of the triangular libration points for the photo gravitational elliptic restricted problem of three bodies, Celestial Mechanics and Dynamical Astronomy, 48, No. 4 (1990), 299-317.

[13] A.P. Markeev, Resonance effects and stability of stationary rotation of a satellite(Motion of dynamically symmetric satellite under action of gravitational moments, discussing stability and nonlinear oscillations, Kosmicheskie Issledovaniia, 5 (1967). 
[14] A.P. Markeev, On one special case of parametric resonance in problems of celestial mechanics, Astronomy Letters, 31, No. 5 (2005).

[15] V.V. Markellos, E. Perdios, and P. Labropoulou, Linear stability of the triangular equilibrium points in the photogravitational elliptic restricted problem, Astrophysics and Space Science, 194, No. 2 (1992).

[16] S.W. McCusky, Introduction to Celestial Mechanics, Addison Wesley (1963).

[17] Manju and R. K. Choudhry, On the stability of triangular libration points taking into account the light pressure for the circular restricted problem of three bodies, Celestial mechanics and dynamical Astronomy, 36, No. 2 (1985).

[18] Carl D.Murray and Stanley F. Dermott, Solar System Dynamics, Cambridge university press, 1999.

[19] A. Narayan and Amit Shrivastava, Existence of resonance stability of triangular equilibrium points in circular case of the planar elliptical restricted three-body problem under the oblate and radiating primaries around the binary system, Advances in Astronomy, 2014 (2014).

[20] A. Narayan and T. Usha, Stability of triangular equilibrium points in the elliptic restricted problem of three bodies with radiating and triaxial primaries, Astrophysics and Space Science, 351, No. 1 (2014).

[21] P.V. Subba Rao and Ram Krishan Sharma, Effect of oblateness on the non-linear stability of L4 in the restricted three-body problem, Celestial Mechanics and Dynamical Astronomy, 65, No. 3 (1996), 291-312.

[22] Victor Szebehely, Stability of the points of equilibrium in the restricted problem, The Astronomical Journal, 72 (1967).

[23] V. Szebehely, Academic press, New-York, 1967.

[24] T. Usha, A. Narayan, and B. Ishwar, Effects of radiation and triaxiality of primaries on triangular equilibrium points in elliptic restricted three body problem, Astrophysics and Space Science, 349, No. 1 (2014), 151-164.

[25] J. Wisdom, A perturbative treatment of motion near the $3 / 1$ commensurability, Icarus, 63, No. 2 (1985).

[26] A.S. Zimovshchikov and V.N. Tkhai, Instability of libration points and resonance phenomena in the photogravitational elliptic restricted three-body problem, Solar System Research, 38, No. 2 (2004). 